\title{
Impacts of deformed bed topography on hydrodynamics in a near-bank vegetated channel: a numerical study
}

\author{
Daoxudong $\mathrm{Liu}^{1,2, *}$, Wenjun $\mathrm{Li}^{2}$ \\ ${ }^{1}$ SAIC Volkswagen; ldxd910712@163.com \\ 2 School of Transportation, Jilin University, China; 1dxd910712@163.com; \\ liwj@mail.jlu.edu.cn \\ * Correspondence: ldxd910712@163.com; Tel.:+86- 17621701712
}

\begin{abstract}
Understanding how the deformed bed topography induced by near-bank vegetation impacts the hydrodynamics is significant for understanding the maintenance condition of bed morphology and further fluvial evolution. This issue has rarely been addressed by current studies. This study with a $2 \mathrm{D}$ hydro-morphological model investigates the hydrodynamics over flat and deformed beds with a near-bank vegetation patch. By varying the patch density, the generalized results show that the hydrodynamics for the deformed bed differs a lot from those for the flat bed. It is found that deformed bed topography leads to an apparent decrease in longitudinal velocity and bed shear stress in the open region and longitudinal surface gradient for the entire vegetated reach. However, the transverse flow motion and transverse surface gradient in the region of the leading edge and trailing edge is enhanced or maintained, suggesting the strengthening of secondary flows. Interestingly, the deformed bed topography tends to alleviate the turbulent effect caused by the junction-interface horizontal coherent vortices, indicating that the turbulence-induced flow mixing is highly inhibited by the deformed bed. Alternatively, the enhanced secondary flows might provide compensation for the flow mixing for the deformed bed, confirmed by a faster recovery of the redistributed water discharge for the vegetated and open regions to the normal value $(50 \%)$. The interior flow adjustment through the patch for the deformed bed requires a shorter distance, which links the vegetative drag length with a logarithmic relation. The tilting bed topographic effect in the open region to accelerate the flow may account for the faster flow adjustment.
\end{abstract}

Keywords: Near-bank patch; Deformed bed topography; Flow adjustment; Hydrodynamics 


\section{Introduction}

Vegetation widely occurs near banks of natural waterways such as rivers, channels and streams. The blockage of vegetation leads the original flow path to the modification, resulting in an evident reduction of flow velocity in the vegetated region but an increase in the adjacent open region $[1,2]$. Sediment transport pattern for the vegetation region is dominated by the depositional process and for the open region by the erosional process. In response to a mobile bed, the bed tends to aggrade for the vegetation region and tends to degrade for the open region. As a consequence, diversity bed forms around the near-bank vegetation are established. These bed forms are identified by pool, riffle and sediment bar [3-5]. Together with the seasonal flow variability, these bed forms providing habitats for different species [6-9] from the perspective of aquatic ecosystem restoration.

For a channel bed occupied by a near-bank vegetation patch, flow mass gets redistributed with more flow is directed to the adjacent open region. As a consequence, the flow in the open region accelerates with velocity increasing and in the vegetated region decelerates with velocity decreasing. The junction of two regions forms significant flow shearing, which under a certain patch density tends to induce flow instability to resemble horizontal coherent vortices with pronounced turbulent activity. The coherent vortices continuously growing along the patch lead to the exchange of flow mass and momentum between the low-velocity vegetated region and high-velocity open region $[1,2,10]$. As the flow exits the vegetated reach, flow separation occurs, leading to the flow in the two regions recovering to the uniform pattern.

When the bed is erodible, bed scour in the open region adjacent to the patch is triggered as flow accelerates or bed shear stress increases. However, suspended loads tend to deposit in the vegetated region for flow deceleration. At equilibrium, the deformed bed topography exerts an effect on the hydrodynamics. For a viewpoint of the same sediment size, the generated scour pool in the open region should alleviate the velocity and bed shear stress to prevent further bed erosion. Correspondingly, other hydrodynamic parameters associated with flow velocity, for instance, water surface gradient and turbulence, should be also influenced by the deformed bed topography. Therefore, the application of the hydrodynamic knowledge of near-bank vegetated flows obtained for a flat bed may be uncertainty for a deformed bed. Numerous studies have demonstrated pronounced impact of bed topography on hydrodynamics under a range of geometric boundaries [11-14]. For instance, Blanckaert (2010) experimentally examine the bed topographic effect on hydrodynamics in a bend channel and found that the deformed bed topography might lead to the enhancement of secondary flows and alleviation of flow shear-induced turbulence. By far, there are rarely studies investigating how the deformed bed topography impacts the hydrodynamics in near-bank vegetated channels either experimentally or numerically. There are studies [13, 15] show how the hydrodynamics behave around a circular patch with deformed bed topography.

This study aims to investigate the difference in flow characteristics around near-bank vegetation over a flat bed or deformed bed. To achieve this goal, a 2D depth-averaged hydro-morphological model is employed to simulate flow and bed adjustment around a near-bank vegetation patch. Specifically, the flow motion is solved by the shallow water equations with the vegetation effect modeled by the drag force method and the bed deformation is solved by an Exner equation with the pre-solved flow fields. One 
advantage of numerical modeling is to conveniently monitor the dynamic change of hydrodynamics and morphodynamics during computation. The co-evolution of hydrodynamics and morphodynamics reflect how the erodible bed immediately responses to the adjustment of hydrodynamics, which are significant issues concerned in this study. The main objective of this study is to investigate the feedback of deformed bed to the hydrodynamics. Therefore, to stay focus, the parameter influencing the bed deformation adopted in the modeling is vegetation density. Other parameters such as patch length, patch width, water discharge and sediment size maintained constant are not included in the analysis.

\section{Mathematical model}

\subsection{Model description}

A 2D depth-averaged hydro-morphological model (Nay2DH, an open source code) is used to simulate flow around a near-bank vegetation patch and bed morphological evolution. This model incorporates a hydrodynamic module and a morphodynamic module, which are explicitly correlated with each other. The hydrodynamic module is the shallow water equations, governing the depth-averaged effect of water flow motion. To model the vegetation blockage effect to the water flow, the vegetation patch is regarded as a porous media and its effect is represented by the drag force method (a quadratic velocity law), which has been widely used in modeling vegetated flow $[1,3,16,17]$. To close the Reynolds stress terms, a $k-\varepsilon$ turbulence model is used to solve the eddy viscosity. The sediment motion and fluvial processes are then solved by the morphodynamic module. With the flow velocities computed by the hydrodynamic module, a bed-load transport equation estimates the sediment flux, which in this study we employ the Meyer-Perter and Muller's formula. The bed deformation is solved by the Exner equation, which links the change in bed elevation to the bed-load flux in the longitudinal and transverse directions. To avoid the non-physic large-gradient bed topography, a slope failure model based on sediment's angle of repose is used for bed deformation correction $[18,19]$.

The governing equations of the flow motion and bed deformation are discretized by the finite difference method. The cubic interpolated pseudoparticle (CIP) method is mainly applied as a third-order numerical scheme, which ensures the accuracy of the numerical solution [18]. For the flow governing equations, at the inlet, the water discharge is specified as the inflow boundary and at the outlet a constant water depth is specified. At the sidewall, the no-slip condition is applied for flow velocities, turbulent kinetic energy and the dissipation rate. For the governing equations of morphodynamics, at the inlet, sediment supply is not given to carry out a clear water scour process which is consistent with the experiment. Detailed mathematical description can refer to Jang and Shimizu [18] and the model manual. 


\subsection{Flume experiment for model verification}

The verification of the hydro-morphological model is conducted by a flume experiment ( $\mathrm{Xu}$ et al. 2019), which demonstrates the bed morphological change around a near-bank vegetation patch as shown in Figure 1. The bed slope is set to $1 / 300$. The near-bank patch with a length of $2 \mathrm{~m}$ occupies a half of the flume bed with a width of $0.31 \mathrm{~m}$. The patch is located in the center region of experimental reach is about $6 \mathrm{~m}$ in length. A layer of sediment with a median size of $1.42 \mathrm{~mm}$ is laid on the flume bottom. The layer thickness is set as $6.5 \mathrm{~cm}$ which is thick enough to ensure the bed being erodible during the entire scour experiment. The vegetation patch is mimicked by an array of solid cylinders, which is rectilinearly arranged. With the cylinder spacing of $0.03 \mathrm{~m} \times 0.03 \mathrm{~m}$, the patch density $a=5.56 \mathrm{~m}^{-1}$. Assuming the drag coefficient $C_{d}=1.2$, the drag length scale $\left(C_{d} a\right)^{-1}=0.15 \mathrm{~m}$. For the experiment, the water discharge $Q=45 \mathrm{~m}^{3} / \mathrm{h}$ and the downstream depth $H_{d}=0.085 \mathrm{~m}$. Therefore, the averaged velocity $U=0.474 \mathrm{~m} / \mathrm{s}$. The experimental setup parameters for the initial condition are summarized in Table 1. To direct the flow from the flat bottom to the sediment bed smoothly, a titling gravel layer was installed in front of the sediment bed. At equilibrium of bed scour, the bed topography was measured by a point gauge in a dense grid. An acoustic Doppler velocimetry (ADV) was used to measure flow velocity over the equilibrium bed topography for different cross-sections as shown in the figure.

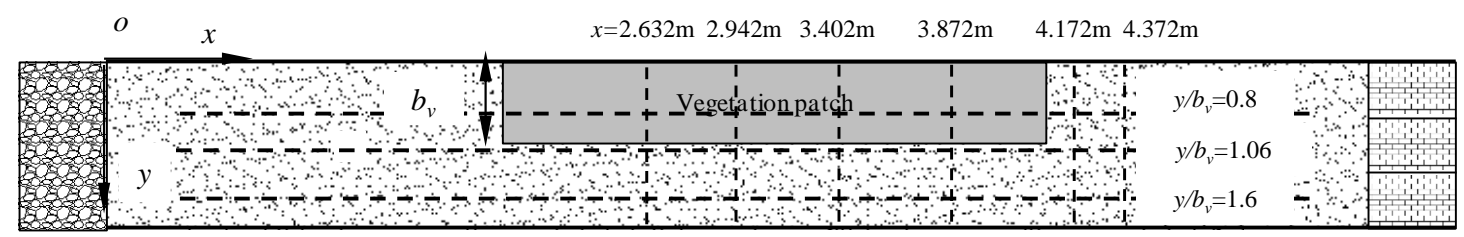

Figure 1 Sketch of flume experiments. The dashed lines indicate the measurement profiles of velocity and bed topography for model verification.

\section{Results}

\subsection{Model verification}

The verification of the depth-averaged hydro-morphological model is conducted by comparing the simulated flow velocities and bed topography with flume data. Figure $2 \mathrm{a}$ presents the verification with respect to the longitudinal velocities along the patch. It is apparent that the simulated flow velocities agree well with the experimental results. The average error ranges from $8 \%$ to $19 \%$, with lower error in the entrance region and higher error in the further-downstream region. This is likely because the flow along the patch gradually evolves with the generation of secondary flows, which impacts the redistribution of flow mass and momentum to lead the difference in error. A 2D numerical model, however, is relatively poor to simulate the effect of secondary 
flows. Figure $2 \mathrm{~b}$ shows the performance in modeling bed deformation. The simulated longitudinal bed profiles fit the measured points. In particular, the matching $\left(y / b_{v}=\right.$ 1.06 and 1.6) is better for the open region. For the vegetated region, the bed degradation for the downstream reach in the vegetated region is underestimated. This might be because the drag force method characterizing only the effect of individual cylinders is not sufficient to describe sediment erosion through individual cylinders. However, the simulated bed deformation for the open region is accurate which draws more attention for research purposes in this study. Therefore, it can be said that the hydro-morphological model can generally give satisfying results, and the model will be used for further analysis of the hydrodynamics over flat and deformed bed topography, which will be emphasized in the following several sections.
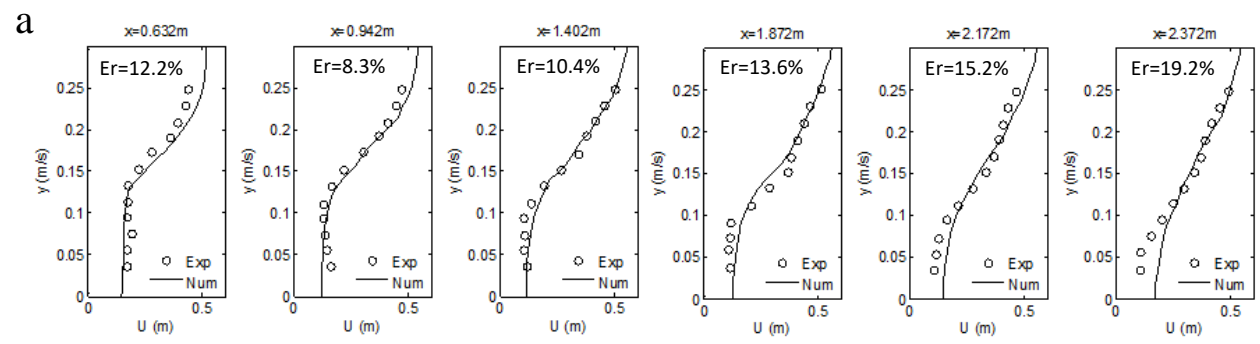

b

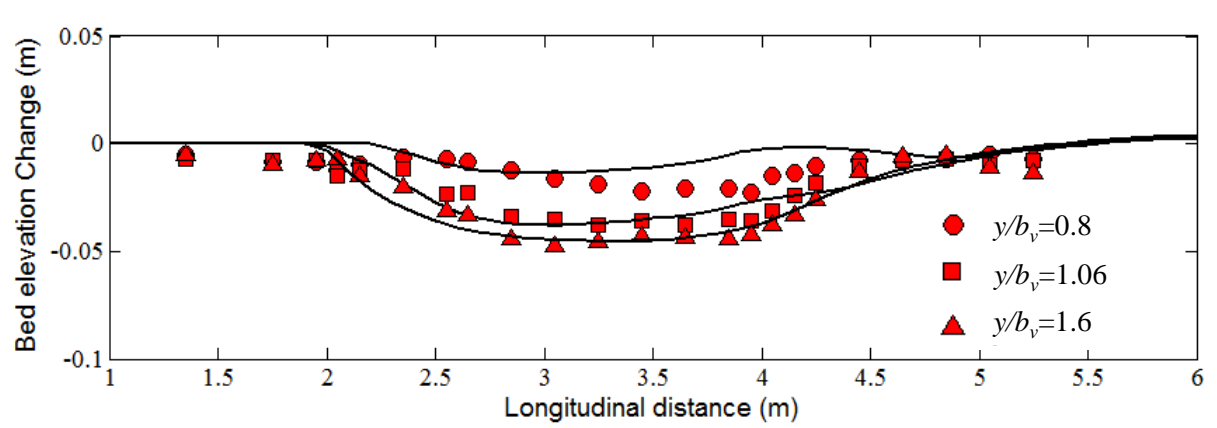

Figure 2 Model verification for the (a) longitudinal bed topography and (b) longitudinal velocity.

\subsection{Bed topography characteristics}

The intention of this study is to explore the impact of bed deformation on hydrodynamics in a near-bank vegetated channel. Therefore, it is better to know the bed topography characteristics under different patch densities. Figure 3 shows that bed topographic evolution under different patch densities starting from the same flat bed. It can be observed that as the patch density increases, the scour pool in the open region adjacent to the patch is produced and enhanced by the accelerating flow velocities and the eroded sediment tends to deposit in the downstream region. The pool enhancement occurs in both the vertical and longitudinal dimensions. For the smallest density $\left(a=1 \mathrm{~m}^{-1}\right)$, the patch exerts a lighter impact on the flow, which as a result leads to a scour pool locating in the far-downstream region with the bed near the entrance less eroded. A significant finding is that as the patch density increases the deepest area of the pool tends to expands upstream, resulting in the elongation of the 
scour pool. However, the longitudinal dimension of the pool is kept comparable to the patch length even if the patch density continuously increases.
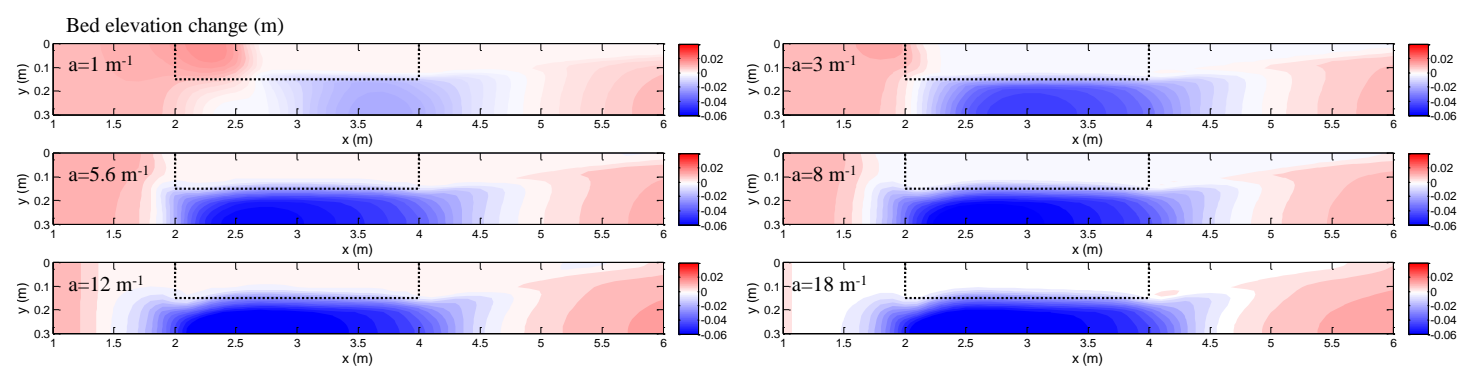

Figure 3 Deformed bed topography around the near-bank vegetation patch under different patch densities

\subsection{Hydrodynamics over deformed bed topography: water surface, flow velocity, bed shear stress and turbulence}

Having known the bed topography characteristics under the increasing patch density, we herein examine how the deformed bed topography impacts the hydrodynamics. The first examined parameters are longitudinal and transverse velocities, which are shown in Figure 4. The longitudinal velocity performs an adjustment after entering the vegetated reach, with flow decelerating in the vegetated region and accelerating in the open region both for the flat bed and deformed bed. However, the bed deformation topography induces a response of the longitudinal velocity differing from that for the flat bed. Over the flat bed, the velocity for the open region continuously increases as flow propagates downstream, with the magnitude peaking in the region of the trailing edge (downstream end). Meanwhile, the velocity magnitude continuously increases with the increasing patch density but the high velocity core continues to expand upstream. Over the deformed bed, the longitudinal velocity distribution is similar to that over the flat bed with a major difference that the longitudinal velocity is significantly relieved compared with that over the flat bed. It can be observed that the longitudinal velocity in the open region has a similar magnitude range even if the patch density increases. The decreasing velocity with the increasing patch density in the vegetated region requires an increasing net discharge in the open region to meet the mass continuity law. The larger cross-sectional area due to the deepening and elongation of the scour pool ensures the increasing net discharge without increasing the velocity. Another phenomenon is that for the deformed bed, a wider transverse transitional region links the low velocity region and high velocity region in contrast to a sharp variation for the flat bed. Moreover, the transitional band is wider for a larger patch density.

For the transverse velocity for the flat bed (Figure $4 \mathrm{~b}$ ), negative and positive zones occur near the leading edge and trailing edge, reflecting the flow convergence and divergence behavior corresponding to the presence of the near-bank patch. The increasing patch density enhances the magnitude of the two zones for the flat bed. For the deformed bed, a more pronounced transverse flow motion can be observed even 
over a minor deformed bed for the smallest patch density. Likewise, a larger patch density induces more enhanced transverse flow motion but the enhancement might be lighter than that for the flat bed. The pronounced transverse motion indicates that the bed deformation may enhance the effect of the secondary flows evolving along the patch, which has been found in the channel occupied by a near-bank patch. Of interest is that for both the flat bed and deformed bed, the positive velocity zone near the trailing edge transforms from a long pattern to a short one as the patch density increases, indicating that the flow separation effect gets more pronounced.
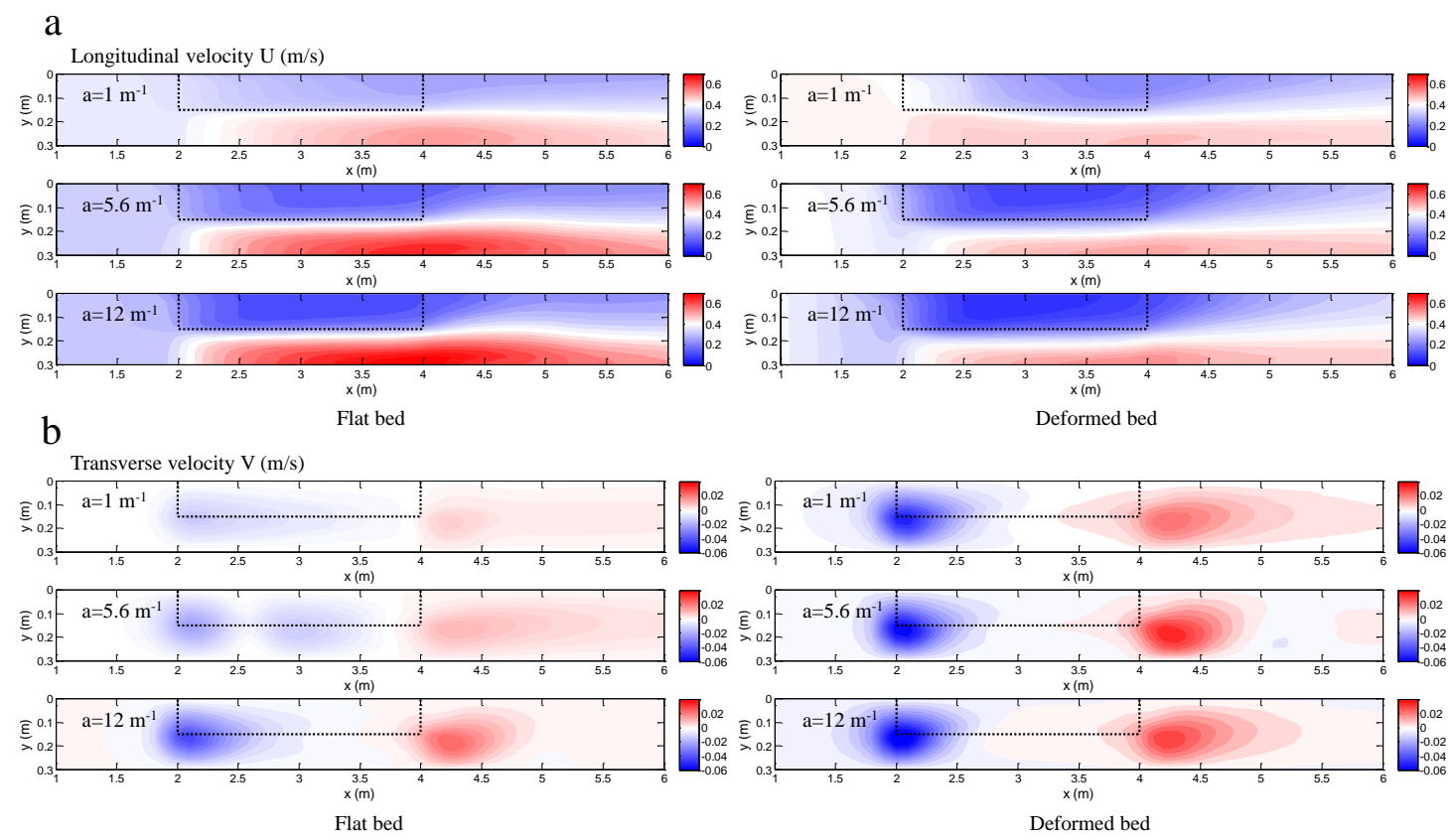

Figure 4 Fully-developed transverse velocity around the near-bank vegetation patch for a flat bed and deformed bed.

Due to the blocking of the vegetation patch, the momentum is redistributed spatially due to the contribution of different hydrodynamic effects. In between, the effect of the hydraulic pressure gradient characterized by the water surface gradient plays an essential role. For the flat bed, the longitudinal surface gradient $\left(S_{x}=\partial z_{s} / \partial x\right)$ becomes pronounced for the vegetated reach (Figure 5a). This is attributed to the outstanding vegetative resistance, which is partially balanced by the force due to the positive hydraulic pressure gradient. Under a small density $\left(a=1 \mathrm{~m}^{-1}\right)$, the water surface gradient tends to be uniform along the patch. As the patch density increases, a higher magnitude core appears in the entrance region of the patch. This might be because the entrance energy work consumed by the patch related to velocity loss is more pronounced for a larger density. Furthermore, the high core diffuses to the adjacent open region with a light decay, which is more pronounced for a larger density. For the deformed bed, the longitudinal water surface gradient is generally lower than that for the flat bed. However, it can be observed that the distribution pattern is similar to that for the flat bed. The tilting bed topography may account for this decay effect. Interestingly, negative value arises in front of the vegetated reach for the deformed 
bed, which is negligible for the flat bed.

Compared with the longitudinal component, the transverse surface gradient $\left(S_{x}=\partial z_{s} / \partial x\right)$ performs similarly for the flat bed and deformed bed (Figure $5 b$ ). At the entrance, a positive-negative zone pair distributes orderly. The first positive zone indicates that the hydraulic pressure may drive transverse flow motion toward the open region and the incipient transverse motion of sediment from the vegetated zone to the open zone. The pattern of the transverse surface gradient well corresponds to the transverse velocity. Downstream the exit, a negative-positive zone pair distributes orderly as well. The first negative zone corresponds to the flow separation in the patch wake, which may also partially contribute to the transverse motion of sediment toward the patch wake. Furthermore, the effect of the transverse surface gradient pattern is enhanced as the patch density increases.
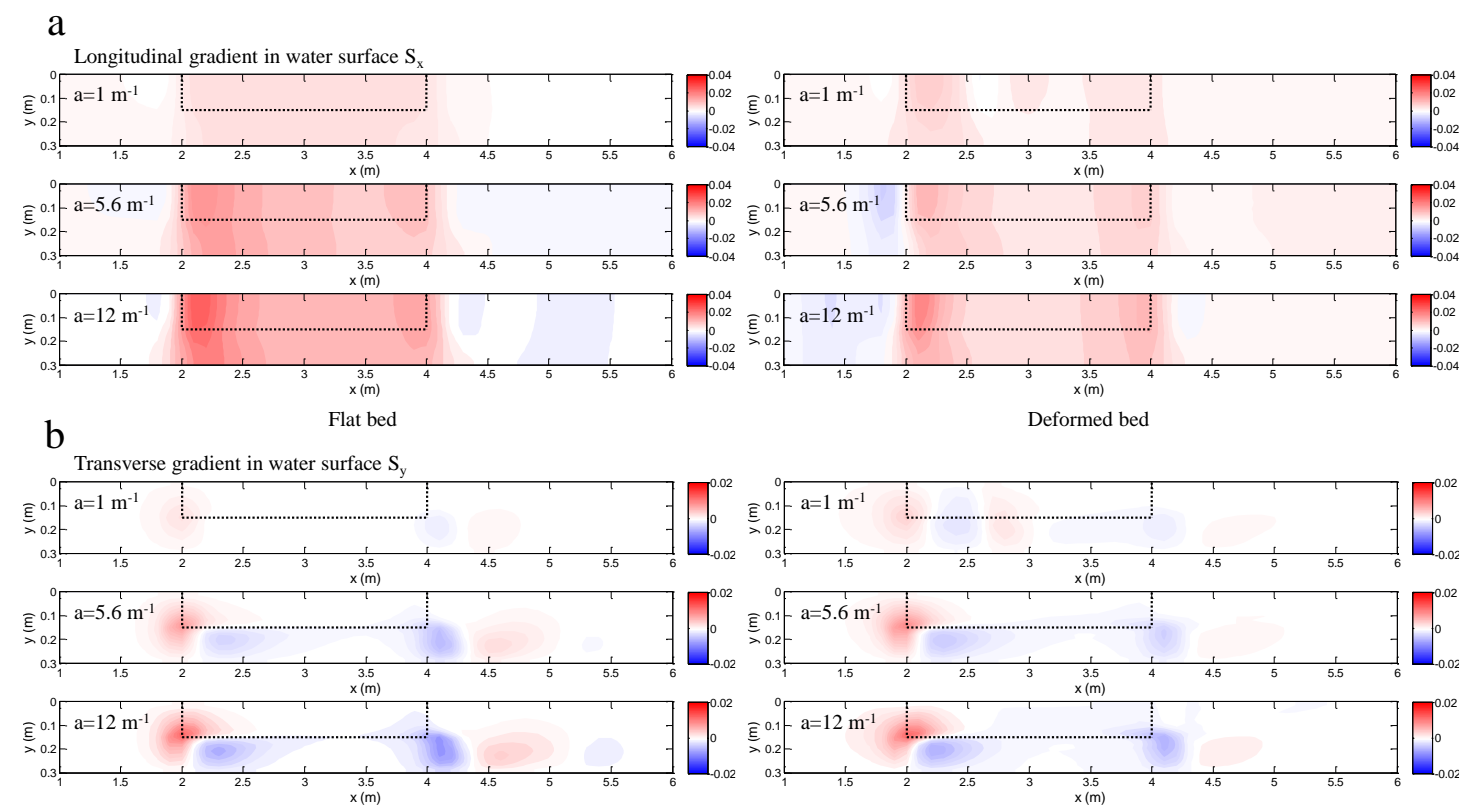

Flat bed

Deformed bed

Figure 5 Surface gradient in the (a) longitudinal and (b) transverse directions around the near-bank vegetation patch for a flat bed and deformed bed.

The distribution of the bed shear stress around the patch impacts the sediment motion and stable bed topography (Figure 6). For the flat bed, the bed shear stress concentrates in the open region near the trailing edge similar to the pattern of longitudinal velocity, indicating the most erodible area. The bed shear stress in the vegetated region decreases greatly due to the addition of vegetation resistance. As the patch density increases, the zone of concentrated bed shear stress tends to expand both upstream and downstream. However, the concentrated zone for the initial condition is not consistent with the orientation of the scour pool at equilibrium under a larger patch density, indicating that the bed shear stress redistributes during the bed topographic adjustment. After the bed topography adjustment is completed, the bed shear stress distribution performs a distinct pattern. The bed shear stress in the vegetated zone only differs slightly from that for the flat bed. This is because the bed 
in the vegetated zone is maintained well by the vegetation without apparent bed deformation. The concentrated bed shear stress in the open region, however, highly decreases as the bed is eroded. This can be explained by that the excessive bed shear stress vanishes as the upper-layer sediment is eroded. A larger patch density still leads to higher bed shear stress. This is because the more tilting bed topography exerting a topographic effect on sediment under a larger patch density requires larger critical shear stress to drive the incipient motion of sediment compared with uniform flow.
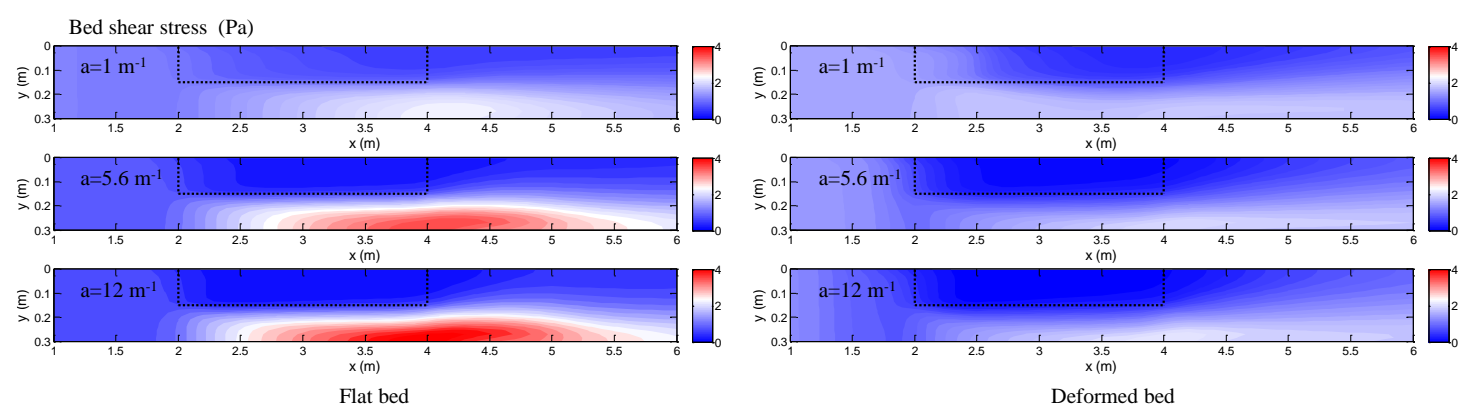

Figure 6 Fully-developed bed shear stress around the near-bank vegetation patch for a flat bed and deformed bed.

The partial blocking of the near-bank patch produced a low-velocity zone in the vegetated region and a high-velocity zone in the open region, which produce a significant flow shear along the interface. The flow shear inducing the flow instability gives rise to horizontal coherent vortices. The vortices can be well quantified by the vorticity $(=\partial U / \partial y-\partial V / \partial x)$, which is shown in Figure 7. For the flat bed, the vorticity arises from the junction interface after the flow entering the vegetated reach and laterally develops along the patch till the trailing edge, indicating continuous development of horizontal coherent vortices. The increasing patch density promoting the flow shearing is likely to produce a pronounced vorticity field. The above results are well consistent with previous studies by both experiments and simulations [20, 21]. For the deformed bed, the spatial vorticity pattern totally changes compared with that for the flat bed. First, it can be found that the magnitude of vorticity for the deformed bed is generally lower than that for the flat bed. This is consistent with the reduced longitudinal velocity for the deformed bed (see Figure 4a). Different from the continuous development of the vorticity along the patch for the flat bed, a wider significant zone of the vorticity is reached near the entrance region and then the significant zone continues to get shrunk along the patch. The transformed pattern of vorticity indicates that the bed deformation tends to alleviate the turbulent extent through reducing the effect of the flow shearing. As compensated, the secondary flows indicated by enhanced transverse flow motion (see Figure 4b) might play an outstanding role in the exchange of mass and momentum. 

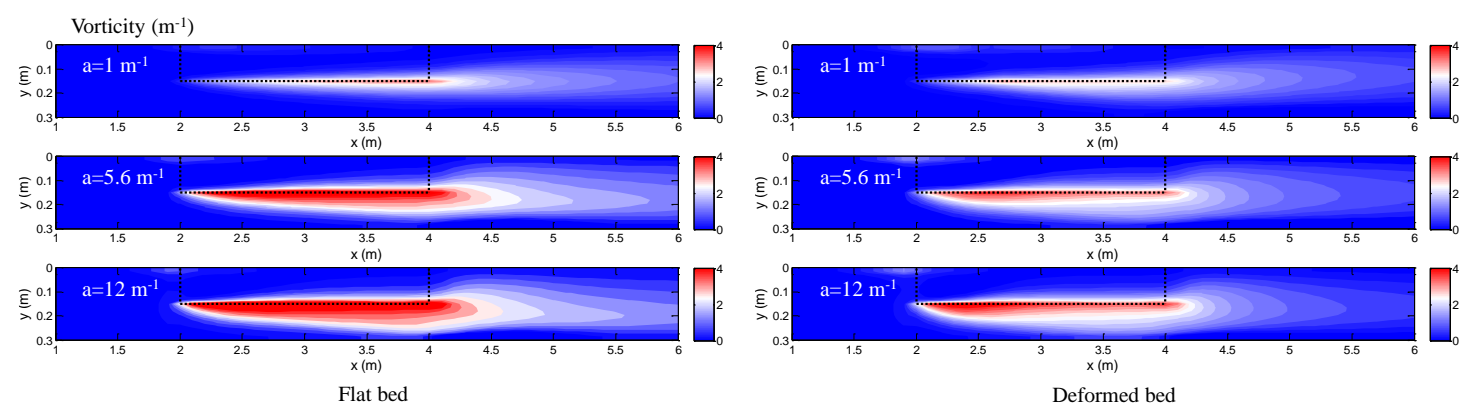

Figure 7 Fully-developed vorticity around the near-bank vegetation patch for a flat bed and deformed bed.

\section{Discussion}

The verified 2D hydro-morphodynamic model allows a deep analysis of the impact of the bed deformation on the hydrodynamics. The presentation of hydrodynamics over the flat bed acting as reference allows a better understanding of the effect of the deformed bed topography. When a patch of vegetation partially occupies the channel bed, the hydrodynamics firstly adjust due to the spatial balance between the individual components of momentum. Flow velocity shows a characteristic pattern regarding the longitudinal velocity and transverse velocity, respectively. The longitudinal velocity decelerates for the vegetated region and accelerates for the open region and peaks near the trailing edge for the flat bed, which is closely related to the distribution of the bed shear stress. The distribution of longitudinal velocity and bed shear stress indicates that the bed erosion in the open region initiates near the trailing edge of the patch. For a small patch density $\left(a=1 \mathrm{~m}^{-1}\right)$, the deepest area of the scour pool is consistent with that of bed shear stress. However, as the patch density increases, the deepest area of the scour pool expands upstream, differing from the patterns of the longitudinal velocity and bed shear stress for the flat bed. Interestingly, the elongated pool profile varies with consistently the distribution of the longitudinal velocity and bed shear stress for the deformed bed, the magnitudes of which however are significantly reduced.

With the bed topography being deformed, the growth in longitudinal velocity in the lateral direction is developed with a larger width at the downstream of the vegetated reach (See Figure 4a); and this effect is enhanced as the pool is deepened by the increase in patch density. This suggests that the lateral development of the longitudinal velocity is attributed to the transverse tilting bed topography. An extreme scenario is that for the flat bed the lateral development of longitudinal velocity has a sharp transition near the junction. For a flat bed, the transverse growth of the longitudinal velocity or flow mixing is impacted by the generated horizontal coherent vortices indicated by vorticity (Nepf [10] stating that for a flat bed horizontal vortices form for $a h>0.1$, with $h=$ water depth). The distribution of the vorticity along the junction for the deformed bed is much lower than that for the flat bed. Therefore, the above statements indicate that the deformed bed through reducing the velocity in the open region diminishes the shear effect (mixing) in the junction region but exerts the 
topographic effect to enhance the transverse flow mixing. The essential cause is that the bed topographic effect might enhance the effect of secondary flow, which is likely to exceed the mixing effect induced by horizontal vortices. The increased depth-averaged transverse velocity around the patch might support this thought (see Figure 4b). Strong secondary flows are commonly present over deformed bed topography such as compound beds $[22,23]$.

The 2D depth-averaged model cannot simulate the generation of the secondary flow evolving along the patch, which has been found in flume experiments and numerical simulations [17, 24]. Some hydrodynamic parameters, however, (for instance, transverse velocity and transverse surface gradient) can indicate its formation. For either a flat bed or deformed bed, pronounced transverse surface gradient occurs as the flow encounters the patch. The positive value indicating hydraulic pressure head tends to drive the transverse flow motion from the patch to the open region, which is confirmed by the negative transverse velocity (see Figure $4 b$ ). As the patch density increases, the surface gradient and transverse motion both become more pronounced so that secondary flows are most likely to be triggered. However, when the bed is deformed, the transverse flow motion near the leading edge is enhanced despite that the surface gradient is reduced. The transverse motion for the deformed bed is negatively contributed by the reduced surface gradient but more positively enhanced by the tilting bed topography. Therefore, the deformed bed topography is expected to induce more intense secondary flows compared with those for the flat bed. The secondary flows induce transverse momentum exchange and thus can promote the flow adjustment.

Previous studies show that the interior adjustment of flow in the vegetated region is related to the blocking effect, characterized by the patch drag length $\left(\left(C_{d} a\right)^{-1}\right)$ and patch width $\left(b_{v}\right)$. Rominger and Nepf [2] based on scale analysis and experimental data proposed a quantitative formula to describe such relation, i.e., $L_{a}=(3.0 \pm 0.3)\left[\frac{2}{C_{d} a} 1+\left(C_{d} a b\right)^{2}\right]$. Likely, it is worth examining how the deformed bed topography impacts the interior flow adjustment. Figure 8 shows that the longitudinal variation of the longitudinal velocity in the vegetated region $\left(y / b_{v}=0.25\right)$ as the patch density increases for the flat bed and deformed bed. The full adjustment of flow is estimated by the velocity varies by less than $5 \%$. It can be clearly observed that for all scenarios the full adjustment of flow (regarding the longitudinal velocity) for the flat bed needs a longer distance $\left(L_{a}\right)$. The increasing patch density (negatively related to $\left.\left(C_{d} a\right)^{-1}\right)$ leads to a further decrease in the distance of fully flow adjustment for both the flat bed and deformed bed, agreeing well with the observed results by previous studies. By plotting the drag length and longitudinal velocity, the two sets of data for the flat bed and deformed bed can be well described by a logarithmic formula, respectively, as shown in the Figure 9. Interestingly, the two formulas differ from each other only by a difference of the intersect of 0.2 . In other words, the interior flow adjustment distance for the deformed bed is shorter than that for the flat bed by an intersect of 0.2 , systematically. However, the obtained relation greatly deviates from 
the proposed formula by Rominger and Nepf [2] in terms of low patch density (high $\left.\left.C_{d} a\right)^{-1}\right)$. This might be because the setting patch length for sparse vegetation is insufficient for the interior full adjustment of flow.
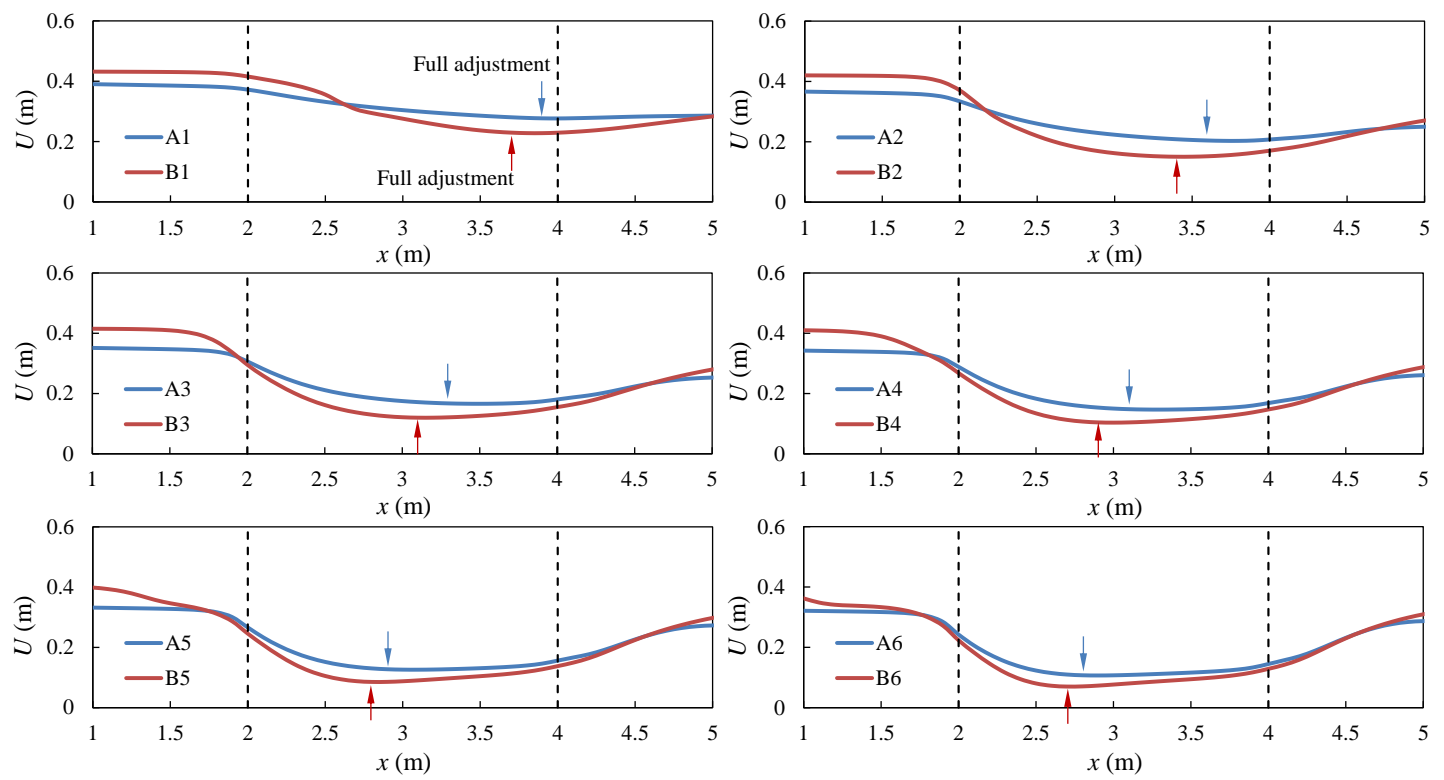

Figure 8 Interior adjustment of the longitudinal velocity in the vegetation region for the flat bed and deformed bed

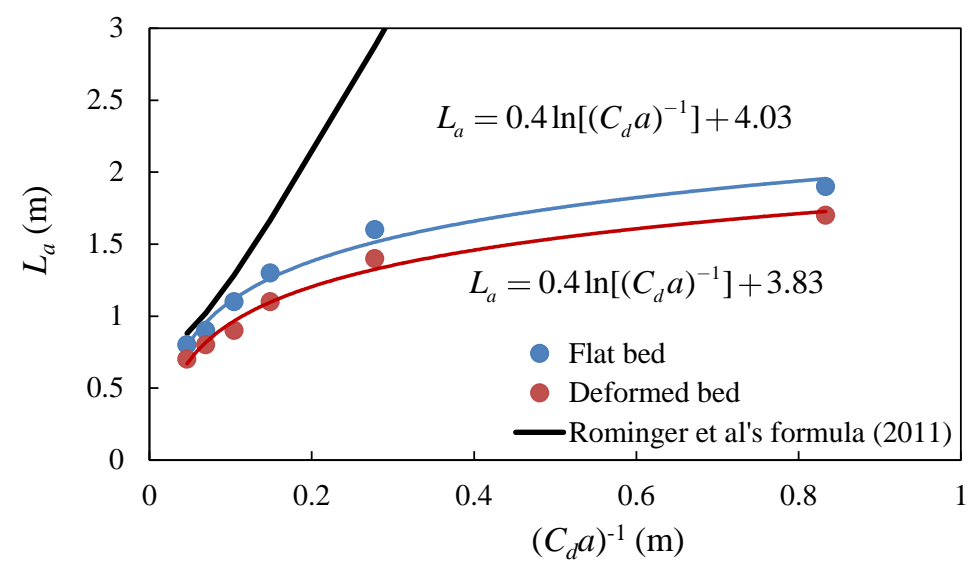

Figure 9 Relation between the flow adjustment distance and drag length for the flat bed and deformed bed

To investigate the redistribution of the water discharge impacted by the near-bank patch may well account for the effect of the deformed bed topography. By integrating the unit discharge (velocity multiplying grid cross-sectional area) over the width for the vegetated region and open region, Figure 10 shows the longitudinal evolution of net discharge along the patch. For both the flat bed and deformed bed, the redistribution of water discharge in the open region and vegetated region requires a longer distance for the patch density decreases, which is consistent with the adjustment of longitudinal velocity. As the bed is deformed, the adjustment discharge is also getting shorter and this is probably because the titling bed topography close to the entrance of the patch accelerates the flow adjustment. Furthermore, water mass is 
more distributed into the open region and less distributed into the vegetation region due to that the enlarged cross-sectional area induced by bed deformation could accommodate more water mass. Of interest is that the onset point of the discharge redistribution occurs further upstream away from the patch as the patch density increases and is more enhanced by more deformed bed topography. Downstream the patch, the deformed bed topography apparently leads the discharge ratio for the open region and vegetated region more quickly to recover to its normal value $(50 \%)$ without the impact of vegetation. This might be also attributed to the generation of secondary flows originating from the vegetated reach (e.g., $x=2 \mathrm{~m}-4 \mathrm{~m}$ ). The secondary flows enable more pronounced flow mixing between the low-velocity flow in the vegetated region and high-velocity flow in the open region, highly exceeding the mixing effect due to pronounced horizontal coherent vortices for the flat bed. The mixing effect of secondary flows can be also reflected by the discharge ratio at the vegetated reach. For a larger density $\left(a=5.6 \mathrm{~m}^{-1}\right.$ and $\left.12 \mathrm{~m}^{-1}\right)$, slightly decaying and growing trends after the full redistribution of the discharge can be observed for the deformed bed, which are not found for the flat bed. The characteristics of discharge redistribution for the vegetated region and open region suggest that the deformed bed (tilting bed topography) accelerates the flow adjustment regarding flow velocity and water discharge both for the vegetated region and open region. The bed deformation-induced secondary flows may enhance the mixing of the high-velocity flow in the open region and low-velocity flow in the vegetated region, particularly facilitating the recovery of the discharge ratio to its normal value $(50 \%)$. 


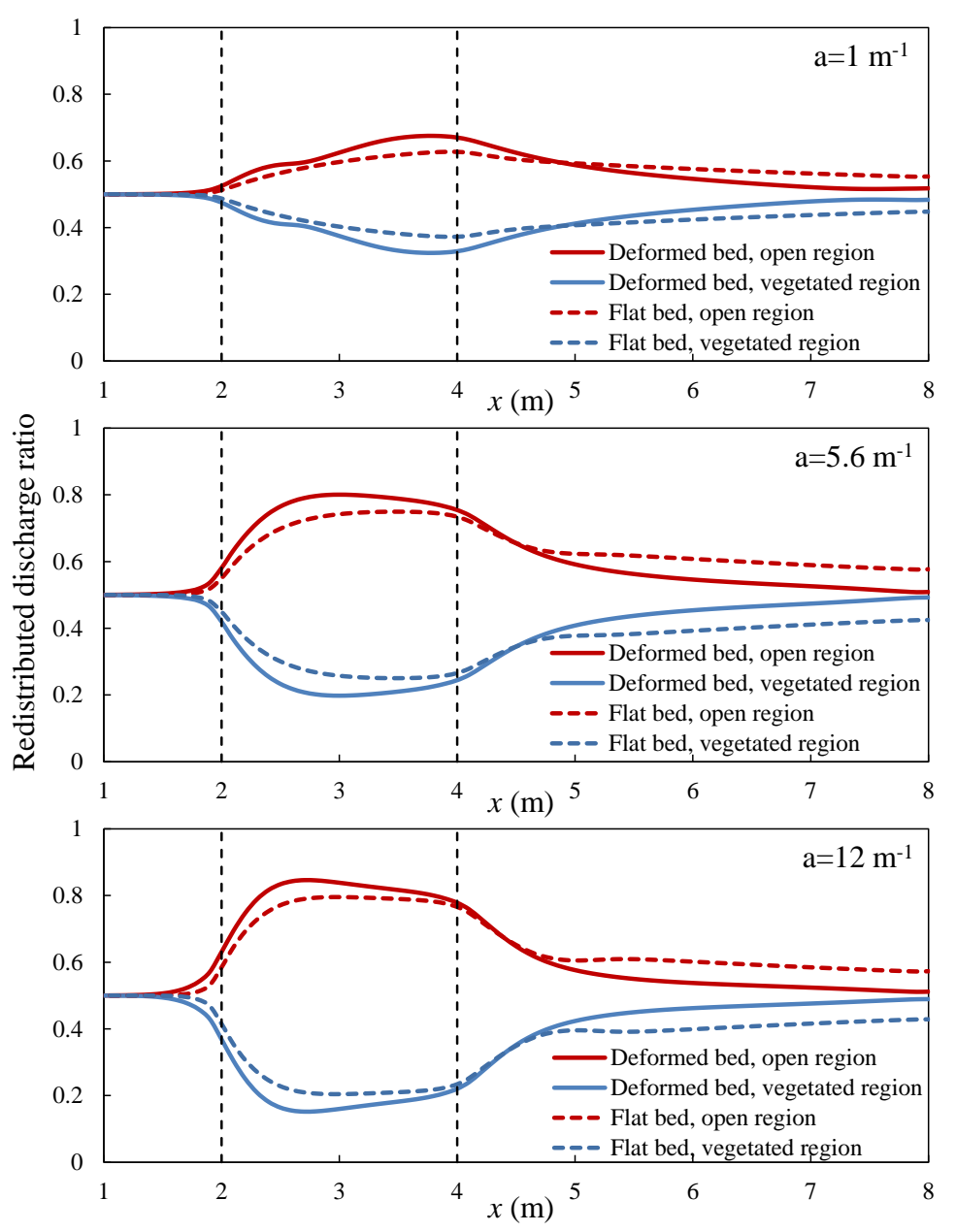

Figure 10 Redistribution of discharge ratio in the open region and vegetated region for the flat bed and deformed bed.

\section{Conclusion}

The growth of near-bank vegetation is likely to induce the adjustment of both hydrodynamics and surrounding bed topography. How the deformed bed topography impacts the hydrodynamics determines the bed stability, future fluvial evolution and ecological effect. Furthermore, the existing knowledge for near-bank vegetated flows for flat beds may bring uncertainty for the deformed bed. However, this important issue has been rarely addressed by the existing studies. The present study conducts a numerical investigation of the impact of the deformed bed topography on the hydrodynamics in an open channel occupied by a near-bank vegetation patch. The strategy of this study is to first verify the 2D hydro-morphological model with the hydrodynamics and topography of a deformed bed in a laboratory flume and to secondly apply the model to conduct bed scour numerical experiments under a variety of patch densities. With the simulated results, a major summary can be drawn as follows.

(1) The simulation shows that the deepest area of the scour pool adjacent to the patch expands upstream as the patch density increases. However, the longitudinal velocity 
and bed shear stress for the flat bed (before the initiation of bed scour) peaking near the trailing edge of the patch indicate, the bed shear stress continuously adjusts during the scour process.

(2) The formation of the deformed bed topography leads to a significant reduction of the longitudinal velocity and bed shear stress. The transverse motion of flow near the leading edge and trailing edge indicating flow convergence and divergence, however, is apparently enhanced, which may strengthen the secondary flows along the patch. The deformed bed topography apparently alleviates the longitudinal water surface gradient but has a lighter effect on the transverse surface gradient.

(3) We investigated the flow adjustment over the deformed bed topography and found that the interior adjustment distance regarding the longitudinal velocity is shortened due to the presence of the bed topography. This phenomenon can be explained by the effect of the tilting bed topography near the entrance of the vegetated reach. A logarithmic relation is found to exist between the adjustment distance and vegetative drag length, which deviates from the formula proposed by previous studies in terms of sparse vegetation. The shortening of flow adjustment distance by the deformed bed topography is also suggested by the redistribution of the net water discharge for the vegetated region and open region.

(4) The vorticity arising from coherent vortices along the junction between the patch and adjacent open region is strongly inhibited by the deformed bed topography compared with that for the flat bed. This indicates that the deformed bed topography can reduce the extent of turbulence and flow mixing between the vegetated region and open region. Alternatively, the secondary flows generated in the open region can compensate for the flow mixing effect of the reduced turbulence.

(5) The strengthened secondary flows can be not only indicated by the increased transverse flow motion near the leading and trailing edges but also indicated by the redistribution of discharge ratio in the vegetated region and open region. The strengthened secondary flows over the deformed bed topography can well explain a faster recovery of the redistributed discharge ratio to a normal value $(50 \%)$ compared with that for the flat bed.

\section{Acknowledgement}

This research is not supported by any founding.

\section{References}

1. Yan X-F, Wai W-HO, and Li C-W (2016) Characteristics of flow structure of free-surface flow in a partly obstructed open channel with vegetation patch. Environmental fluid mechanics. 16(4): 807-832.

2. Rominger JT and Nepf HM (2011) Flow adjustment and interior flow associated with a rectangular porous obstruction. Journal of Fluid Mechanics. 680: 636-659. 
3. Xu Z-X, Ye C, Zhang Y-Y, Wang X-K, and Yan X-F (2019) 2D numerical analysis of the influence of near-bank vegetation patches on the bed morphological adjustment. Environmental Fluid Mechanics: 1-32.

4. Bennett SJ, Wu W, Alonso CV, and Wang SS (2008) Modeling fluvial response to in - stream woody vegetation: implications for stream corridor restoration. Earth Surface Processes and Landforms: The Journal of the British Geomorphological Research Group. 33(6): 890-909.

5. Kim HS, Kimura I, and Shimizu Y (2015) Bed morphological changes around a finite patch of vegetation. Earth Surface Processes and Landforms. 40(3): 375-388.

6. Willis S, Winemiller K, and Lopez-Fernandez H (2005) Habitat structural complexity and morphological diversity of fish assemblages in a Neotropical floodplain river. Oecologia. 142(2): 284-295.

7. Santos JM, Branco P, Katopodis C, Ferreira T, and Pinheiro A (2014) Retrofitting pool-and-weir fishways to improve passage performance of benthic fishes: effect of boulder density and fishway discharge. Ecological Engineering. 73: 335-344.

8. Xu M-z, Wang Z-y, Pan B-z, and Na Z (2012) Distribution and species composition of macroinvertebrates in the hyporheic zone of bed sediment. International Journal of Sediment Research. 27(2): 129-140.

9. Zhao N, Wang ZY, Pan BZ, Xu MZ, and Li ZW (2015) Macroinvertebrate assemblages in mountain streams with different streambed stability. River Research and Applications. 31(7): 825-833.

10. Nepf HM (2012) Hydrodynamics of vegetated channels. Journal of Hydraulic Research. 50(3): 262-279.

11. Blanckaert K (2010) Topographic steering, flow recirculation, velocity redistribution, and bed topography in sharp meander bends. Water Resources Research. 46(9).

12. Koken M and Constantinescu G (2011) Flow and turbulence structure around a spur dike in a channel with a large scour pool. Water Resources Research. 47(12).

13. Chang W-Y, Constantinescu G, and Tsai WF (2017) On the flow and coherent structures generated by a circular array of rigid emerged cylinders placed in an open channel with flat and deformed bed. Journal of Fluid Mechanics. 831: $1-40$.

14. Konsoer KM, Rhoads BL, Best JL, Langendoen EJ, Abad JD, Dan RP, and Garcia MH (2016) Three-dimensional flow structure and bed morphology in large elongate meander loops with different outer bank roughness characteristics. Water Resources Research. 52(12): 9621-9641.

15. Gu J, Shan Y, Liu C, and Liu X (2018) Feedbacks of flow and bed morphology from a submerged dense vegetation patch without upstream sediment supply. Environmental Fluid Mechanics,(8).

16. Zeng C and Li CW (2014) Measurements and modeling of open-channel flows with finite semi-rigid vegetation patches. Environmental Fluid Mechanics. 
14(1): 113-134.

17. Choi S-U and Kang H (2006) Numerical investigations of mean flow and turbulence structures of partly-vegetated open-channel flows using the Reynolds stress model. Journal of Hydraulic Research. 44(2): 203-217.

18. Jang C-L and Shimizu Y (2005) Numerical simulation of relatively wide, shallow channels with erodible banks. Journal of Hydraulic Engineering. 131(7): 565-575.

19. Fischer-Antze T, Stoesser T, Bates P, and Olsen N (2001) 3D numerical modelling of open-channel flow with submerged vegetation. Journal of Hydraulic Research. 39(3): 303-310.

20. White BL and Nepf HM (2007) Shear instability and coherent structures in shallow flow adjacent to a porous layer. Journal of Fluid Mechanics. 593: $1-32$.

21. Huai W, Xue W, and Qian Z (2015) Large-eddy simulation of turbulent rectangular open-channel flow with an emergent rigid vegetation patch. Advances in Water Resources. 80: 30-42.

22. Yang SL, Zhang J, and Xu X (2007) Influence of the Three Gorges Dam on downstream delivery of sediment and its environmental implications, Yangtze River. Geophysical Research Letters. 34(10).

23. Kang $\mathrm{H}$ and Choi S-U (2006) Turbulence modeling of compound open-channel flows with and without vegetation on the floodplain using the Reynolds stress model. Advances in Water Resources. 29(11): 1650-1664.

24. Nezu I and Onitsuka K (2001) Turbulent structures in partly vegetated open-channel flows with LDA and PI V measurements. Journal of hydraulic research. 39(6): 629-642. 